\title{
East Kent IT collaborative for healthcare (E-KITCH) - responding to the pandemic through optimising use of information technology
}

\author{
Author: Mahdi Abdulla ${ }^{\mathrm{A}}$
}

\section{Introduction}

The Never too busy to learn guidance released in April 2020 highlights the benefits of having trainees at the forefront of quality improvement (QI) through digital means. The COVID-19 pandemic led to major disruption in the traditional delivery of services. The East Kent IT Collaborative for Healthcare (E-KITCH) was established to facilitate trainee participation in a number of projects ranging from enhanced handover to QR code posters and education. We demonstrate the success in one such project delivering online medical education following the introduction of social distancing measures.

\section{Methods}

All initiatives exclusively used systems available to the trust. To this end, Webex was used as the standardised medium of delivery. A 'how to' session was set up to orient and educate senior clinicians, followed by the delivery of education sessions across all sites within the trust. Starting in May 2020, cardiology teaching followed including COVID-themed radiology teaching and journal clubs. Data collection was digitised using Google Forms. A 21-point questionnaire was compiled and sent out to trainees via various trust WhatsApp groups and NHSMail mailing lists.

A bespoke E-KITCH 'Recipe Box' consisting of PowerPoint presentation slides and a Wiki format 'QI Cookbook' to help guide trainees and facilitate further trust-wide QI initiatives were also provided. Further links were made between IT, medical education and audit so as to formally recognise efforts from within the trust and ensure complain with local information governance.

\section{Results}

A total of 18 responses were collected.

$>100 \%$ reported no issues or very easy access with the Webex platform.

> $76.9 \%$ of responders either agreed or strongly agreed with the statement 'the Webex platform allowed ease of interaction with my instructor and colleagues that is comparable to that of a regular classroom:

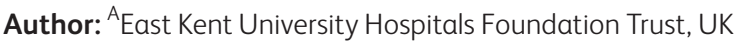

$>80.7 \%$ agreed with the statement that 'a blended approach with online teaching help fit around my schedule'.

$>88.5 \%$ found the course material to be very easy to view on the Webex platform.

\section{Discussion}

The success of this was followed by successful implementation of junior doctors' induction. The COVID-19 pandemic has highlighted the need to optimise use of IT systems available to the NHS. This study highlights the of one such example in east Kent which was led by trainees.

With no archive of trust-wide QI tools, available efforts were made to establish one within the trust. Further work will need to be done to evaluate and refine the QI tools available.

As a generation of 'digital natives', it validates guidance by the RCP that having a formal process to facilitate IT-based QI has brought significant benefits to the trust while also nurturing leadership and a sense of autonomy among trainees and junior doctors.

Much more scope for improvement lies with the news that NHS organisations must implement new terms with Microsoft 365 by completing a participation agreement by October 2021. In addition to the security benefits this provides, this also offers the benefits of an NHS-wide directory for identity management and access. ${ }^{2}$

\section{Conclusion}

This demonstrates the success of IT-centred QI strategy. With an expanding landscape of digital tools available to NHS organisations, its serves as a framework from which to facilitate and incentivise trainees to integrate services and push boundaries of healthcare innovation.

\section{Conflicts of interest}

None declared.

\section{References}

1 Royal College of Physicians. Never too busy to learn: How the modern team can learn together in the busy workplace. London: RCP, 2018.

2 NHS Digital. Microsoft Office 365 for the NHS. NHS, 2020. https:// digital.nhs.uk/services/microsoft-office-365-for-the-nhs. [Accessed 01 October 2020]. 